\title{
A Case of Multiple Recurrences of Trigeminal Neuralgia: Attributed to Central Sensitization?
}

\section{Yuankun Cai}

Wuhan University Zhongnan Hospital https://orcid.org/0000-0003-1563-3006

\section{Xiuling Zhang}

Wuhan University Zhongnan Hospital

Xuan Dai

Wuhan University Zhongnan Hospital

\section{Songshan Chai}

Wuhan University Zhongnan Hospital

\section{Bangkun Yang}

Wuhan University Zhongnan Hospital

\section{Bing Shu}

Wuhan University Zhongnan Hospital

\section{Mengyang Wang}

Wuhan University Zhongnan Hospital

\section{Guo Li}

Wuhan University Zhongnan Hospital

Wen Liu

Wuhan University Zhongnan Hospital taojunjin Lu

Wuhan University Zhongnan Hospital

\section{Zhixin Zheng}

Wuhan University Zhongnan Hospital

\section{Zhimin Mei}

Wuhan University Zhongnan Hospital

Yixuan Zhou

Wuhan University Zhongnan Hospital

Jingwei Zhao

Wuhan University Zhongnan Hospital Jingyi Yang

Wuhan University Zhongnan Hospital

\section{Lei Shen}

Wuhan University Zhongnan Hospital Joshua Ho 
School of Biomedical, LKS Faculty of Medicine, Hongkong university

Jincao Chen

Wuhan University Zhongnan Hospital

Kui Liu

Wuhan University Zhongnan Hospital

Nanxiang Xiong ( $\sim$ mozhuoxiong@163.com )

Wuhan University Zhongnan Hospital

\section{Case report}

Keywords: Trigeminal Neuralgia, Central Sensitization, Trigger Point, Recurrence

Posted Date: June 11th, 2021

DOl: https://doi.org/10.21203/rs.3.rs-593228/v1

License: (c) (1) This work is licensed under a Creative Commons Attribution 4.0 International License. Read Full License 


\section{Abstract}

Background Although many hypotheses about the pathogenesis of trigeminal neuralgia have been developed, there is little discussion about the multiple recurrences of trigeminal neuralgia.

Case presentation Here we report a patient with refractory multiple recurrent trigeminal neuralgia, in which we accordingly found that an increase in facial pain trigger points and a rapid movement of the upper limb extremity could trigger an onset of trigeminal neuralgia. We offer hypothesis on the multiple recurrences of trigeminal neuralgia by explaining the cause of multiple trigger points in this patient.

Conclusions Given that central sensitization is involved in a number of mechanisms of pain development and is associated with some of the symptoms of trigeminal neuralgia. We hypothesized that central sensitization may have been involved in the disease development of this case of refractory trigeminal neuralgia, which led to the development of trigger point generalization and multiple recurrence after surgical treatments.

\section{Background}

With the development of magnetic resonance imaging (MRI) and microneurosurgical techniques, the recurrence rate of trigeminal neuralgia $(\mathrm{TN})$ has decreased significantly[3, 11, 13]. However, there are still some patients who have multiple recurrences and which aren't adequately explained. We report a case with multiple recurrent TN who present multiple trigger points and a new onset trigger point, with facial pain triggered by rapidly moving both upper limbs. We refer to this phenomenon as trigger point generalization and hypothesize that it is associated with multiple recurrences in the patient.

Central sensitization is a pain hypersensitivity response caused by central neuroplasticity, which is associated with many of the symptomatic features and therapeutic effects of acute and chronic pain[12, $16,18]$. And it was suggested that central sensitization may also be participating in the pathogenesis of $\operatorname{TN}[2,9,19]$. Therefore, we propose a hypothesis: central sensitization may be related to the disease development of this case of refractory $\mathrm{TN}$, which lead to the development of trigger point generalization and multiple TN recurrence.

\section{Case Presentation}

A 52-year-old male patient suffered from paroxysmal facial pain for 21 years. The patient described this pain as intense like an electric shock, concentrated on the right side of the mandible and the perioral area, and lasting only seconds or minutes before spontaneous relief. The patient had a prominent trigger point on the right side of the mandible, and facial pain was triggered by touching the skin in this area or by brushing the teeth. After 14 years of conservative treatment with carbamazepine early in the course of the disease, the frequency of facial pain attacks gradually increased and required increasingly higher doses of carbamazepine. In 2014, the patient underwent the first microvascular decompression, after which the symptoms were relieved. However, the patient's disease later recurred and he underwent another 
microvascular decompression and a gamma knife treatment at 2 different hospitals in 2016 and 2017, respectively. There was a period of remission after both procedures, but symptoms soon recurred.

Three years ago, the patient came to our hospital with a recurrence of trigeminal neuralgia. On physical examination, we found multiple trigger points on the patient's face, but this did not attract enough attention because there were cases of multiple trigger points in previous experience and in the literature. The patient was discharged after treatment with trigeminal ganglion balloon compression. The patient came back to our hospital 6 months ago with a recurrence of trigeminal neuralgia, and this time we made a new discovery: the classic trigeminal neuralgia could be trigger by a rapid movement of the both upper limbs. The patient underwent brain MRI, which reveal tight adhesion of the fifth cranial nerve to the adjacent structures (Fig. 1); hence trigeminal ganglion balloon compression was carried out. The patient was effectively relieved at postoperative follow-up, but it would still be important for us to follow up for recurrence.

\section{Discussion And Conclusions}

A popular explanation for the pathogenesis of $\mathrm{TN}$ is the ignition hypothesis[7, 15]. According to the hypothesis, TN is attributed to a specific abnormality of the trigeminal afferent neurons in the trigeminal roots or ganglia. Light stimulation from the trigger zone causes a paroxysm of pain by causing synchronized post-discharge activity in the hyperexcitable afferent neurons[7]. However, the hypothesis cannot explain how the posterior discharge is caused by stimulation from zones extending beyond the trigeminal branch dominion. Therefore, we have to think that there are other mechanisms involved in the formation of trigger point generalization and multiple TN recurrence.

The concept of central sensitization was first introduced by Woolf[20]. It refers to the increased membrane excitability and synaptic efficacy of somatosensory neurons established in the dorsal cortex of the spinal cord after intense peripheral noxious stimulation, tissue damage, or nerve injury, lead to decrease of threshold, overreaction to stimulus and the spread of nociceptive field [6, 9]. Central sensitization is responsible for neuropathic pain[10,21], migraine[4], postinjury pain[5] and fibromyalgia[8, 17], and is associated with some symptoms of trigeminal neuralgia[14, 19]. Therefore, we hypothesized that central sensitization may be associated with trigger point generalization and promote TN recurrence. Patients with recurrent TN suffer from intense facial pain for periods of time, which provides an ideal condition for the formation of central sensitization. Once central sensitization is established, the increased membrane excitability and synaptic efficacy of primary neurons in the spinal trigeminal nucleus leads the patients to exhibit nociceptive hyperalgesia as well as trigger point generalization.

If this hypothesis is to hold true, it could explain to some extent the clinical features of the patient in our report. As with other TN patients, typical facial pain can be triggered by light touching through the regular trigeminal sensibility pathway (Fig. 2A). After central sensitization, however, a seemingly irrelevant action

could also activate the sensitive spinal nucleus of trigeminal nerve in some way, thus triggering the same 
facial pain (Fig. 2B). At the same time, patients with central sensitization had multiple recurrences of TN due to an increased chance of triggering facial pain as a result of trigger point generalization.

The presence of trigger points as a TN diagnostic criterion is present in almost all patients, which are most commonly present in the perioral and mandibular regions $[1,7,10]$. Previous studies have reported that patients with TN may have abnormal trigger points $[8,13]$, but recurrent TN presenting with new abnormal trigger points has not been reported. We report and discuss a case of multiple recurrent TN with multiple trigger points and propose the hypothesis, hoping that it will be of help to the diagnosis and treatment of patients with recurrent TN. Of course, more experimental and clinical studies are needed to explore the causes of recurrence in patients with $\mathrm{TN}$.

\section{Abbreviations}

MRI: magnetic resonance imaging

TN: trigeminal neuralgia

\section{Declarations}

Funding: Not applicable.

Conflicts of interest/Competing interests: The authors declare no competing interests.

Availability of data and material: Data may be given upon reasonable request.

Code availability: Not applicable.

Ethics approval: Consent from the patient is obtained.

Consent to participate: All authors have reviewed the manuscript and approved its publication.

Consent to publication: All authors read and approved the final version of the manuscript.

\section{Authors' contributions:}

Concept and design: Kui Liu and Nanxiang Xiong.

Data collection and analysis: all authors.

Original draft preparation: Yuankun Cai, Xiuling Zhang and Xuan Dai.

Manuscript review and editing: all authors.

Supervision: Kui Liu and Nanxiang Xiong. 
All authors reviewed and commented on previous versions of the manuscript.

\section{Acknowledgments}

The authors would like to thank Jiayi Chen for her assistance in generating Fig. 2

\section{References}

1. (2018) Headache Classification Committee of the International Headache Society (IHS) The International Classification of Headache Disorders, 3rd edition. CEPHALALGIA 38

2. -211

3. Ayzenberg I, Obermann M, Nyhuis P, Gastpar M, Limmroth V, Diener HC, Kaube H, Katsarava Z (2006) Central sensitization of the trigeminal and somatic nociceptive systems in medication overuse headache mainly involves cerebral supraspinal structures., pp 1106-1114

4. Barker FGN, Jannetta PJ, Bissonette DJ, Larkins MV, Jho HD (1996) The long-term outcome of microvascular decompression for trigeminal neuralgia. N Engl J Med 334:1077-1083

5. Burstein R, Yarnitsky D, Goor-Aryeh I, Ransil BJ, Bajwa ZH (2000) An association between migraine and cutaneous allodynia. ANN NEUROL 47:614-624

6. Campbell JN, Raja SN, Meyer RA, Mackinnon SE (1988) Myelinated afferents signal the hyperalgesia associated with nerve injury. PAIN 32:89-94

7. Cook AJ, Woolf CJ, Wall PD, McMahon SB (1987) Dynamic receptive field plasticity in rat spinal cord dorsal horn following C-primary afferent input. NATURE 325:151-153

8. Devor M, Amir R, Rappaport ZH (2002) Pathophysiology of trigeminal neuralgia: the ignition hypothesis. CLIN J PAIN 18:4-13

9. Gracely RH, Petzke F, Wolf JM, Clauw DJ (2002) Functional magnetic resonance imaging evidence of augmented pain processing in fibromyalgia. Arthritis rheumatism 46:1333-1343

10. Hu W, Zhang K, Zhang J (2010) Atypical trigeminal neuralgia: A consequence of central sensitization? MED HYPOTHESES 75:65-66

11. Koltzenburg M, Scadding J (2001) Neuropathic pain. CURR OPIN NEUROL 14:641-647

12. Liu D, Shi P, Li K, Guo Y, Liu X, Wang C, Liu Y, He B, Zhang X (2020) Preoperative visualization of neurovascular contact with 3D-FIESTA combined with 3D-TOF MRA to guide microvascular decompression surgery planning. JOURNAL OF NEURORESTORATOLOGY 8:104-113

13. Nijs J, Torres-Cueco R, van Wilgen CP, Girbes EL, Struyf F, Roussel N, van Oosterwijck J, Daenen L, Kuppens K, Vanwerweeen L, Hermans L, Beckwee D, Voogt L, Clark J, Moloney N, Meeus M (2014) Applying modern pain neuroscience in clinical practice: criteria for the classification of central sensitization pain. PAIN PHYSICIAN 17:447-457

14. Nurmikko TJ, Eldridge PR (2001) Trigeminal neuralgia-pathophysiology, diagnosis and current treatment. Br J Anaesth 87:117-132 
15. OBERMANN M, YOON MS, ESE D, MASCHKE M, KAUBE H, DIENER HC, KATSARAVA Z (2007) Impaired trigeminal nociceptive processing in patients with trigeminal neuralgia. NEUROLOGY 69:835-841

16. Rappaport HZ, Devor M (1994) Trigeminal neuralgia: the role of self-sustaining discharge in the trigeminal ganglion. PAIN 56:127-138

17. Sanchis MN, Lluch E, Nijs J, Struyf F, Kangasperko M (2015) The role of central sensitization in shoulder pain: A systematic literature review. SEMIN ARTHRITIS RHEU 44:710-716

18. Staud R (2002) Evidence of involvement of central neural mechanisms in generating fibromyalgia pain. CURR RHEUMATOL REP 4:299-305

19. van Griensven H, Schmid A, Trendafilova T, Low M (2020) Central Sensitization in Musculoskeletal Pain: Lost in Translation? J Orthop Sports Phys Ther 50:592-596

20. Watson JC (2007) From paroxysmal to chronic pain in trigeminal neuralgia: implications of central sensitization. NEUROLOGY 69:817-818

21. Woolf CJ (1983) Evidence for a central component of post-injury pain hypersensitivity. NATURE 306:686-688

22. Woolf CJ, Mannion RJ (1999) Neuropathic pain: aetiology, symptoms, mechanisms, and management. Lancet 353:1959-1964

\section{Figures}




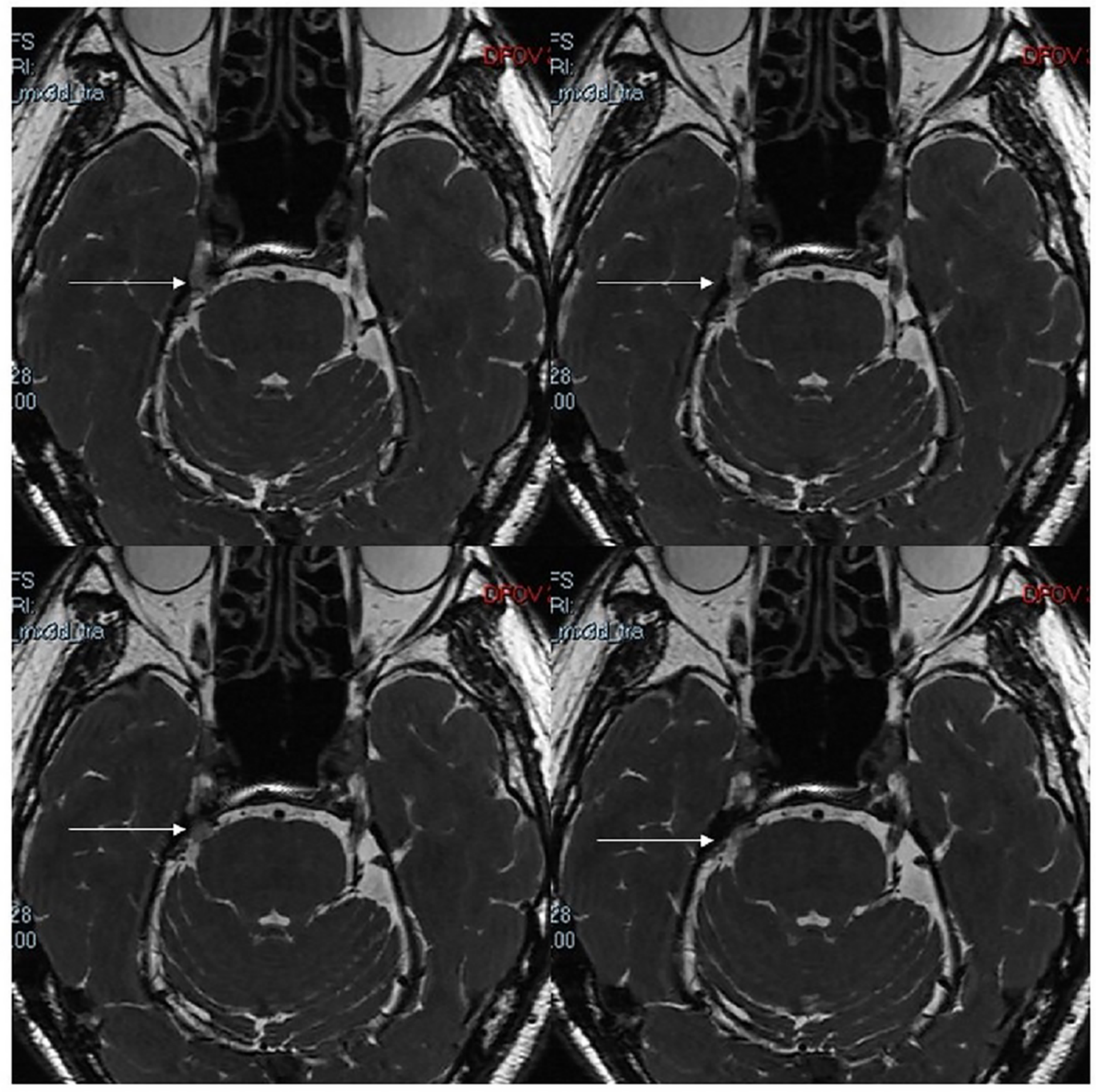

Figure 1

MRI showing tight adhesion of the right fifth cranial nerve to the adjacent structures (white arrow). 


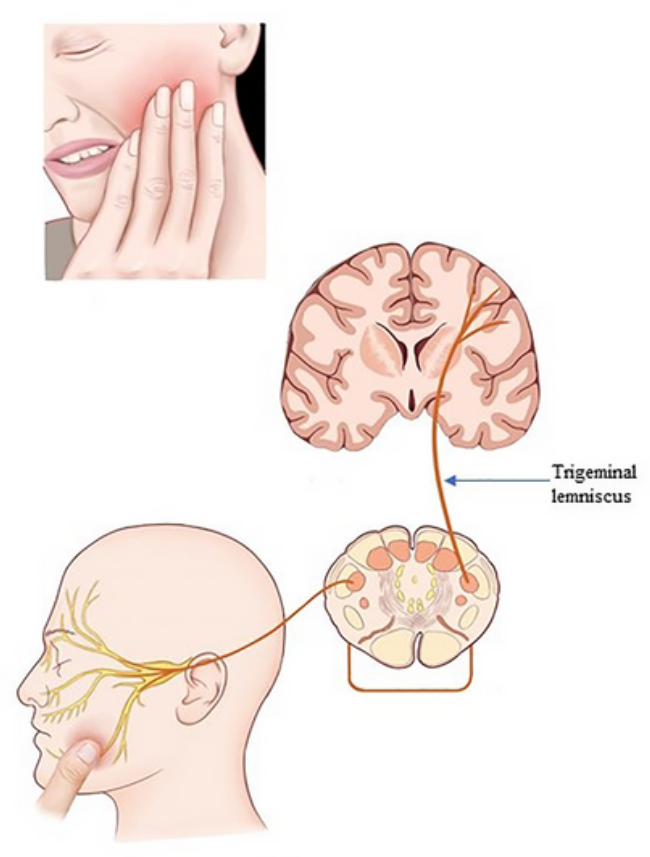

A

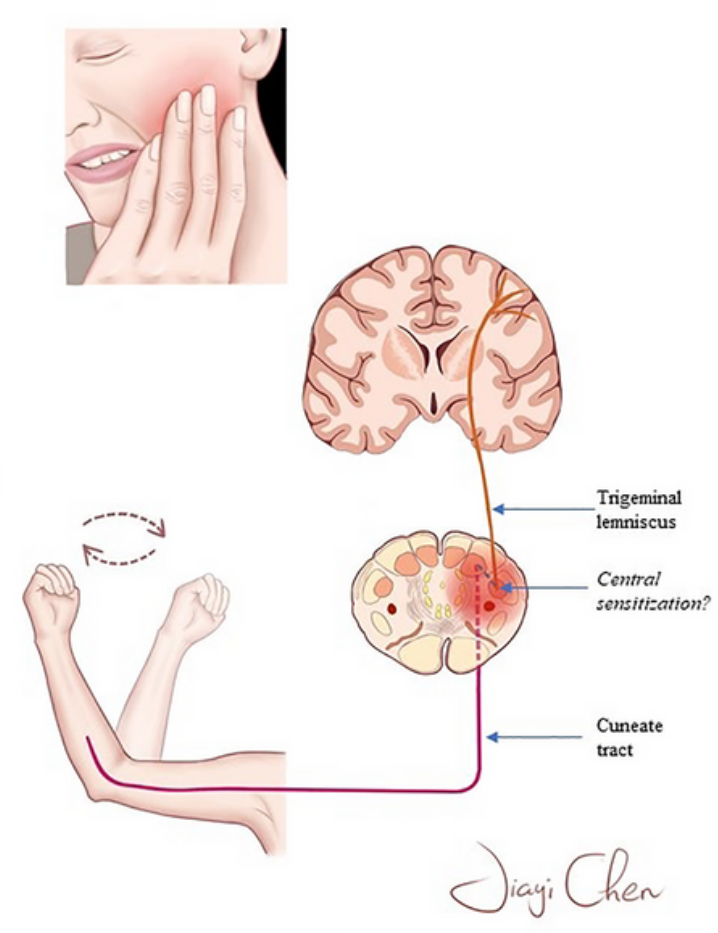

B

\section{Figure 2}

The left schematic diagram (A) shows the conventional pathway of facial pain triggered by lightly touching the corners of the mouth through the trigeminal sensibility pathway. The right schematic (B) shows the pathway of facial pain triggered by shaking the upper limb in some way to activate the sensitive spinal nucleus of the trigeminal nerve after the formation of central trigeminal sensitization. 